\title{
Neonatal Mechanical Ventilation-Early Experiences in central India
}

\author{
Dutt RD', Dutt C ${ }^{2}$, Ambey $\mathbf{R}^{3}$ \\ ${ }^{1}$ Dr R D Dutt, Associate Professor, Department of Pediatrics, ${ }^{2}$ Dr. Chandrakala Dutt, Assistant Professor, Department of \\ Surgery, ${ }^{3}$ Dr. Ravi Ambey, Assistant Professor, Department of Pediatrics. All are affiliated with Kamla Raja Hospital \& G \\ R, Medical College, Gwalior, MP, India
}

Address for correspondence: Dr R D Dutt, Email: drrddutt@rediffmail.com

\begin{abstract}
Introduction: This innovative technology of mechanical ventilation had been started since 1960s and reached to significant level in affluent nation but in our nation, it is in nascent stage. This is first kind of study measuring outcome of neonatal mechanical ventilation in central part of India. Methodology: Study was conducted for one year after permission from Institutional Research board at Neonatal intensive care unit (NICU), of Kamla Raja Hospital, G.R. Medical College, Gwalior (M.P.). It was a prospective observational study \& statistically, SSSP-10 system was applied for study. In our study, we used time cycled, pressure limited, continuous flow ventilator (DRAGGER-8000). Out of total 1160 admission during study period over one year in our NICU, 79 newborns $(6.81 \%)$ requiring mechanical ventilation were enrolled. 70 (88.60\%) newborn were given SIMV mode and 9 (11.40\%) were given exclusive CPAP as per their indication. Results: The overall survival rate was $48.10 \%$ (SIMV $42.85 \%$ (20 cases) and CPAP $88.88 \%$ ( 8 cases) respectively. The inborn babies were $35.44 \%$ (28 cases) and out born were $64.56 \%$ (51 cases) but survival was much better in inborn babies $64.28 \%$ (18 cases) compared to out born babies $39.21 \%$ (20 cases) which is statistically significant $(\mathrm{p}<0.05)$. Though male neonates were predominant $64.55 \%$ (51 cases), but outcome was better in female newborns $57.14 \%$ survival than Male. Conclusion: Mechanical ventilation is in evolving phase in central part of India. In hands training \& experience will further improve outcome after few decades.
\end{abstract}

Key words: CPAP, Mechanical ventilation, Ventilator, Neonate

\section{Introduction}

Mechanical ventilation can be noninvasive or invasive. In today's world every country is competing equally to provide better health care services to their citizen. Even developing countries have achieved a great success in reducing mortality rates and providing good health care system. A great impact of such facilities can be observed from maternal mortality rate (MMR). As in our country we have implemented many programmes to reduce MMR $\&$ provide better $\mathrm{MCH}$ (Mother and Child health) service as a result of it we have drastically reduced MMR from 407 in 1997 to nearly around 301 to 2003 as a result of this more maternal life have been saved and due to this the number of preterm and complicated deliveries turning in successful one have been increased so we are facing a new challenge to our health care system as Neonatal Mortality rate. Through we have achieved a great success in reducing Neonatal Mortality rate to around 37 in 2006. Although we have achieved so many millstones in our health service but

Manuscript received: $1^{\text {st }}$ May 2014

Reviewed: $20^{\text {th }}$ May 2014

Author Corrected: $23^{\text {rd }}$ May 2014

Accepted for Publication: $10^{\text {th }}$ June 2014 still this high NMR when compared with western data is a big road block for our progress.

This innovative technology of mechanical ventilation had been started since 1960s and reached to significant level in affluent nation but in our national it is in only nascent stage. But due its high cost, expert skill requirement has limited its use in developing countries. Though this sophisticated technology has started around 1990s in India. This is first kind of study measuring outcome of mechanically ventilated neonates in India, in this study, we would like to report our experience with neonatal mechanical ventilation or up on 79 sick newborns required mechanical ventilation.

\section{Material and methods}

This is a prospective observational study conducted at Neonatal Intensive Care Unit, Kamla Raja Hospital, Gwalior (M.P.) over a period of one year i.e. from August 2009 to July 2010. We have included both inborn and outborn neonates. Out of total 1160 admission during 
study period over one year in our NICU,79 newborn requiring mechanical ventilation were enrolled after taking written informed consent of parents and also explaining ethical issues. 70 newborn were given SIMV mode and 9 were given exclusive CPAP perinatal history, associated risk factors, indications for mechanical ventilation were recorded on Performa. Major surgical anomalies (e.g. TEF, TGA etc) and parents not given consent were excluded.

Newborn were ventilated as per standard protocol babies were nursed under servo control open care system. Continuous clinical monitoring of Heart Rate, Respiratory Rate SPo2 temp., ECG, NIBP and Blood gases were done every 12 hourly in stable babies or even more frequently in unstable babies and also with each change in ventilator settings blood glucose reading were taken by glucometer sepsis work up done regularly and endotracheal aspirate were sent for suspected pneumonia blood culture was sent for suspected septicemia Chest Xrays were taken for suspected air leak.

Neonates were weaned off on clinical and radiological improvement and normal blood gases values with minimum ventilator support Dexamethasone were given prior to extubation was done for $>72$ hours. Babies were kept under oxygen hood after extubation. Outcomes were analysed for different variables like gestational age birth weight, indications, maternal risk factors and complications. All the observations were analyzed and appropriate statistical tests were applied on observations.

Inclusion Criteria: Sick newborns admitted in NICU, $\mathrm{KRH}$, Gwalior having indications of mechanical ventilation.

\section{Exclusion Criteria:}

1. Preterm $<30$ weeks with Small for G.A.

2. Newborn weighing $<1000 \mathrm{Gms}$.

3. Terminal newborns with major surgically uncorrected lethal anomalies (TEF, TGA etc.)

4. Parent not willing or not given consent for ventilation. 5. Newborns ventilated $<12$ hours.

\section{Aims and objectives:}

1. To assess the various indications, course of illness and outcomes in mechanically ventilated newborns.

2. To observe mortality rates in sick mechanically ventilated newborns.

3. To study various complications in mechanically ventilated newborns.

4. To assess various factors affecting outcome in mechanically ventilated neonates.

\section{Observation}

During study period of one year 79 (6.81\%) admitted babies required mechanical ventilation were enrolled in our study.

Table No 1: Distribution and Outcome According to Sex

\begin{tabular}{|l|l|l|}
\hline & No. of cases & Survived \\
\hline Male & $51(64.55 \%)$ & $22(43.13 \%)$ \\
\hline Female & $28(35.44 \%)$ & $16(57.14 \%)$ \\
\hline Total & 79 & 38 \\
\hline
\end{tabular}

During study period male newborn were $65 \%$ but outcome was better in female newborns. \{Female $57.14 \%$ (16 cases) than Male $43.13 \%$ (22 cases) $\}$

Table No 2: Distribution and Outcome According To Mural Status

\begin{tabular}{|l|l|l|}
\hline Indications & No. of cases & Survival \\
\hline Inborn & $28(35.44 \%)$ & $18(64.28 \%)$ \\
\hline Outborn & $51(64.56 \%)$ & $20(39.21 \%)$ \\
\hline P-Value & & $\mathrm{P}<0.05$ \\
\hline Total & 79 & 38 \\
\hline
\end{tabular}

Inborn babies were $35.44 \%$ (28 cases) and out born were $64.56 \%$ (51 cases) of total population but survival was much better in inborn babies $64.28 \%$ (18 cases) compared to out born babies $39.21 \%$ (20 cases) which is statistically significant ( $\mathrm{p}<0.05)$. 
Table No 3: Distribution and Outcome According To Birth Weight

\begin{tabular}{|l|l|l|}
\hline & No. of cases & Survived \\
\hline $1000-1499 \mathrm{gm}$ & $13(16.45 \%)$ & $5(38.46 \%)$ \\
\hline $1500-2000 \mathrm{gm}$ & $19(24.05 \%)$ & $8(42.10 \%)$ \\
\hline $2000-2500 \mathrm{gm}$ & $15(18.98 \%)$ & $7(46.66 \%)$ \\
\hline$>2500 \mathrm{gm}$ & $32(40.50 \%)$ & $18(56.25 \%)$ \\
\hline Total & 79 & 38 \\
\hline
\end{tabular}

Survival rate increases with increase in gestational age.

Table No 4: Distribution and outcome according to gestational age

\begin{tabular}{|l|l|l|}
\hline Gestational age & No. of cases & Survived \\
\hline$<32$ weeks & $13(16.45 \%)$ & $4(30.76 \%)$ \\
\hline $32-34$ weeks & $16(20.25 \%)$ & $6(37.5 \%)$ \\
\hline $34-37$ weeks & $15(18.98 \%)$ & $8(53.33 \%)$ \\
\hline$>37$ weeks & $35(44.30 \%)$ & $20(57.14 \%)$ \\
\hline Total & 79 & 38 \\
\hline
\end{tabular}

Preterm <37 wks, Term 37 to 42 wks, Post term 42 wks or more

Table No 5: Distribution and outcome according to indication

\begin{tabular}{|l|l|l|}
\hline & No. of cases & Survived \\
\hline Sepsis & $22(27.84 \%)$ & $8(36.36 \%)$ \\
\hline MAS & $18(22.78 \%)$ & $11(61.11 \%)$ \\
\hline HMD & $15(18.98 \%)$ & $8(53.33 \%)$ \\
\hline Asphyxia & $10(12.65 \%)$ & $4(40.00 \%)$ \\
\hline Apnoea of Prematurity & $6(7.59 \%)$ & $3(50.00 \%)$ \\
\hline Aspiration Pneumonia & $4(5.06 \%)$ & $3(50.00 \%)$ \\
\hline
\end{tabular}

The most common indication for ventilation was septicemia-27.84\% (22 cases) followed by hyaline membrane disease$18.98 \%$ (9 cases), birth asphyxia-12.65\% (10 cases), apnea of prematurity-7.59\% (6 cases), aspiration pneumonia-5.06\% (4 cases) and Persistent Pulmonary Hypertension of newborns- 5.06\% (4 cases).

The overall survival rate was 48.10\% (SIMV 42.85\% (20 cases) and CPAP $88.88 \%$ (8 cases). Among maternal factor mother's with previous neonatal losses, Eclampsia/ Pre-eclampsia has poor survival and having mortality as $69.24 \%(9$ cases) \& $60.00 \%$ (3 cases) respectively. The best survival among indication was seen in babies with MAS $61.11 \%$ (11 cases) and HMD-53.33\% (15 cases). Babies with birth asphyxia, septicemia and PPHN have survival as $40 \%$ (4 cases), $36.36 \%$ ( 8 cases), $25 \%$ ( 1 cases) respectively. Complications were septicemia $31.64 \%$ (25 cases), tube block-15.18\% (15 cases), pneumothorrax-7.59\% (6 cases), ventilator associated pneumonia-5.06\% (4 cases), IVH-5.06\%(4 cases) weaning failure-3.79\% (3 cases) and pulmonary Hemorrhage- $2.53 \%$ ( 2 cases).

\section{Discussion}

Neonatal mechanical ventilation has improved survival rates of sick newborns in NICU. In this study, we have given assisted ventilation to 79 babies out of 1160 admission (6.81\%). Nangia et al [1], Mathur et al [2], and Riyas et el [3] reported $8.9 \%, 13 \%$ and $5.6 \%$ babies required mechanical ventilation. This may be due to different factors like draining area, inclusion and exclusion criteria, infrastructure and different admission policies.

Overall survival rate in our study was $48.10 \%$ which is comparable to other Indian studies conducted till now whose survival rates vary from $41.17 \%$ to $55.8 \%$. In CPAP group $88.88 \%$ survived which can be compared with $90 \%$ survival of CPAP group in Krishnan et al [4] 
and $100 \%$ survival in Nangia et al study [1]. In SIMV group we found $42.85 \%$ survival, which also vary from $32.7 \%$ to $42.18 \%[3,6]$. Though most babies, we have given assisted ventilation were out born (64.56\%) compared to inborn (35.44\%), but outcome was much better in intramural babies $(64.28 \%)$ compared to extramural babies (39.21\%), which is statistically significant. This may be due to early intervention and availability of ventilation for inborn babies.

In present study female babies have better survival rate $57.14 \%$ compared to male babies $43.13 \%$ though we found male babies needed more assisted ventilation. Most other Indian Studies also showed similar pattern and this may be contributed to female babies are biologically strong. Among maternal factor's mother's with previous neonatal losses and Eclampsia/Preeclampsia were having poor survival and have mortality rates as $69.24 \%$ \& $60.00 \%$ respectively. Mortality in mother's with previous neonatal losses may be due to various factors like genetic disorder, Maternal factor like Pregnancy induced Hypertension.

The commonest indication in our study was septicemia, followed by Meconium aspiration syndrome, Hyaline membrane disease, birth asphyxia, apnoea of prematurity, aspiration pneumonia and PPHN. Krishnan et al [4], Ruchi rai et al [5] also reported sepsis as their most common indication for ventilation however Singh et al [6], and Maiyya et al [7] reported HMD as most common indication. Riyas et al [3] reported birth asphyxia as most common indication. Low incidence of HMD babies for ventilation can be attributed to more liberal use of antenatal steroids in mothers of anticipated premature babies.

In this study one or more complication were developed in $46.83 \%$ babies. Major complications were septicemia (31.64\%), tube block (15.18\%) pneumothorax (7.59\%). Septicemia was the major complication in other studies as singh et al reported 37\%, Mathur et al [2] reported $36.7 \%$, Riyas et al [3] reported $38.2 \%$ and Nangia et al [1] reported $26 \%$. Septicemia is still a major complication in ventilated babies due to frequent intervention like blood gases and prolonged duration of ventilation. Least possible intervention, minimum possible duration of ventilation and judicious use of antibiotics may reduce its incidence.

Tube block was seen in $15.18 \%$ cases in our study compared to $32.3 \%$ in riyas et al [3] and only $5.8 \%$ in Krishnan et al [4] reported. This lower incidence can be due to frequent endotracheal toilet. Pnuemothorax was seen in only $7.59 \%$ of cases which is comparable with Mathur et al [2] $6.7 \%$ and Krishnan et al [4] 8.8\% respectively. Singh et al [6] (13\%) Riyas et al [3] (13.7) reported higher incidences. Intensive monitoring and early intervention may prove life saving. Ventilator associated pneumonia incidence was quite low in our study having $5.06 \%$ compared to $30.6 \%$ reported by Tripathi et al [8], 28.8\% by Mathur et al [2] and $25 \%$ by Maiyya et el[7]. This can be attributed to careful attention to endotracheal toilet, frequent suctioning, adequate pressure setting and minimum possible duration of ventilation, well established nursery and well trained staff.

Though neonatal mechanical ventilation has effective role in reducing mortality in sick newborn but still we are lagging behind the international data as provided by H.Tortman [9]. Complications cannot be overlooked as it significantly affects the outcome but intensive monitoring and early intervention can reduce its incidence as reported by Lindorth [10]. Care should be taken for barotraumas and minimum possible intervention to avoid septicemia.

\section{Conclusion}

Overall $48.10 \%$ of newborns requiring mechanical ventilations for various indications survived. Out born babies have poor survival compared to inborn babies. Babies of mother with previous fetal loss \& eclampsia has poor outcome. Out study also reconfirms that survival rate increase with birth weight and gestational age. Pnemothorax and VAP were leak complications. Early recognition of complication related with Ventilator support, frequent monitoring \& good nursing care are keys of successful weaning of any neonate.

\section{Funding: Nil \\ Conflict of interest: Nil \\ Permission from IRB: Yes}

\section{References}

1. Nangia S, Saili A, Dutta AK, Gaur V, Singh M, Seth A, Kumari S. Neonatal mechanical ventilationexperience at a level II care centre. Indian J Pediatr 1998 Mar-Apr;65(2):291-6.

2. N.C. Mathur, Sailesh Kumar, A.L. Prasanna, U.K. Sahu, R. Kapoor, S. Roy, Rajiv Chandra and Y.C. Mathur. Intermittent positive pressure ventilation in a neonatal intensive care unit. Indian Pediatrics - April 1998; 35:(1) 4-7 
3. Riyas PK, Vijaykumar KM, Kulkarni ML. Neonatal mechanical ventilation. Indian J Pediatr 2003 Jul; 70(7): 537-40.

4. Krishnan L, Francis PP, D’Souza NA, Bhaskaranand N. Assisted ventilation in neonates : the Manipal experience. Indian J Pediatr 1994 Jul-Aug; 61(4):379-86.

5. Ruchi Rai and D.K. Singh. An Experience with Neonatal Ventilation in Eastern U.P.; Indian Journal of Pediatrics. November 2009;70(1):65-70

6. Singh M, Deorari AK Paul VK Mittal M, Shankar Munshi $U$ et al. three years experiences with neonatal ventilation from tertiary care hospital in delhi. Indian pediatrics 1993 ; 30: 783-789.

7. P.P. Maiya, D. Viswanath, S. Hegde, T.P. Srinivas, Shivprasad, C.C. Shantala, P. Umakumaran, Naveen B.
And R.K. Hegde, Mechanical ventilation of new borns: experience from a level-II NICU 1275 Indian Pediatrics 1995, 32:1-12

8. Dr. Shalini Tripathi, Dr. G.K. Malik, Dr. Amita Jain and Dr. Neera Kohli; Study of Ventilator Associated Pneumonia in Neonatal Intensive Care Unit. characteristics, risk factors and outcome. Internet Journal of Medical Update 2010 January; 5(1): 12-19.

9. H Tortman. The neonatal intensive care unit at the University Hospital of the West Indies. the first few years' experience; West Indian med.j- Mar 2006; 55 (2): 32-36.

10. Lindorth M., Svenningsen, N.W. Ahlstrom, H. and Jonson, B. Evaluation of mechanical ventilation in newborn infants. Techniques and survival rates. Acta Paediatr Scand -1980; 69: 143-149.

\section{How to cite this article?}

Dutt RD, Dutt C, Ambey R. Neonatal Mechanical Ventilation-Early Experiences in central India. Int J Med Res Rev 2014;2(4):319- 323. doi:10.17511/ijmrr.2014.i04.10 